\title{
Treat-to-target (T2T) recommendations for gout
}

\author{
U Kiltz, ${ }_{1}^{1}$ J Smolen, ${ }^{2}$ T Bardin, ${ }^{3}$ A Cohen Solal, ${ }_{1}^{4} \mathrm{~N}$ Dalbeth, ${ }^{5} \mathrm{M}$ Doherty, ${ }_{1}^{6}$ B Engel, ${ }_{1}^{7}$ \\ C Flader, ${ }^{1} \mathrm{~J}$ Kay ${ }_{1}^{8} \mathrm{M}$ Matsuoka, ${ }^{9} \mathrm{~F}$ Perez-Ruiz, ${ }^{10} \mathrm{G}$ da Rocha Castelar-Pinheiro, ${ }^{11}$ \\ $\mathrm{K}$ Saag, ${ }^{12} \mathrm{~A} \mathrm{So}^{13}{ }^{13} \mathrm{~J}$ Vazquez Mellado, ${ }^{14} \mathrm{M}$ Weisman, ${ }^{15} \mathrm{~T} \mathrm{H}$ Westhoff, ${ }^{16}$ \\ H Yamanaka, ${ }^{17}$ J Braun $^{1}$
}

\section{Handling editor Tore K Kvien}

- Additional material is

published online only. To view please visit the journal online (http://dx.doi.org/10.1136/ annrheumdis-2016-209467)

For numbered affiliations see end of article.

Correspondence to

Professor Juergen Braun, Rheumazentrum Ruhrgebiet, Herne D-44652 Germany; juergen.braun@ elisabethgruppe.de

Received 1 March 2016 Revised 26 August 2016 Accepted 27 August 2016 Published Online First 22 September 2016

\section{Q Corsinatk}

To cite: Kiltz U, Smolen J, Bardin T, et al. Ann Rheum Dis 2017;76:632-638.

\section{ABSTRACT}

Objectives The treat-to-target (T2T) concept has been applied successfully in several inflammatory rheumatic diseases. Gout is a chronic disease with a high burden of pain and inflammation. Because the pathogenesis of gout is strongly related to serum urate levels, gout may be an ideal disease in which to apply a T2T approach.

Our aim was to develop international T2T recommendations for patients with gout.

Methods A committee of experts with experience in gout agreed upon potential targets and outcomes, which was the basis for the systematic literature search. Eleven rheumatologists, one cardiologist, one nephrologist, one general practitioner and one patient met in October 2015 to develop T2T recommendations based on the available scientific evidence. Levels of evidence, strength of recommendations and levels of agreement were derived.

Results Although no randomised trial was identified in which a comparison with standard treatment or an evaluation of a T2T approach had been performed in patients with gout, indirect evidence was provided to focus on targets such as normalisation of serum urate levels. The expert group developed four overarching principles and nine T2T recommendations. They considered dissolution of crystals and prevention of flares to be fundamental; patient education, ensuring adherence to medications and monitoring of serum urate levels were also considered to be of major importance. Conclusions This is the first application of the T2T approach developed for gout. Since no publication reports a trial comparing treatment strategies for gout, highly credible overarching principles and level D expert recommendations were created and agreed upon.

\section{INTRODUCTION}

Gout is caused by deposition of monosodium urate (MSU) crystals within joints in the setting of chronic hyperuricaemia. ${ }^{1}$ It affects $1 \%-2 \%$ of adults in developed countries and is the most common type of inflammatory arthritis worldwide. Epidemiological data are consistent with a rise in the prevalence and incidence of gout. ${ }^{2}{ }^{3}$ Nutrition and genetic polymorphisms of renal transporters of urate and other genes seem to be the main causal factors of primary gout. Gout and hyperuricaemia are associated with hypertension, diabetes mellitus, metabolic syndrome and renal and cardiovascular diseases. ${ }^{1}{ }^{4} 5$ There is a strong link between gout and increased risk of death from all causes and cardiovascular diseases. ${ }^{6}$ The economic burden of gout is considerable.

Gout is a common clinical problem encountered by both general and specialist clinicians. The key principles of gout management include establishing a definitive diagnosis, treating acute attacks promptly and using urate-lowering therapies (ULTs) appropriately to dissolve MSU crystals to eventually prevent further attacks and joint damage. When serum urate concentrations are lowered below the saturation point of MSU, new crystal formation is prevented, existing crystals dissolve and gout can be cured. The 'gold standard' diagnostic test for gout remains the identification of MSU crystals by polarised light microscopy in synovial fluid cells or in a tophus.

Non-steroidal anti-inflammatory drugs (NSAIDs) and colchicine remain the most widely recommended systemic drugs to treat acute attacks. ${ }^{9}{ }^{10} \mathrm{In}$ some patients, especially those with comorbidities that preclude NSAID use, oral glucocorticoids (GCs) may represent an alternative. Joint aspiration and injection of GC is recommended where feasible, as in most hospital settings. ${ }^{11-13}$ Patient education, appropriate advice regarding lifestyle and treatment of comorbidities are important in the management of patients with gout. ${ }^{14}$

Many different recommendations on the management of gout have been published over the last decades. ${ }^{11-13}{ }^{15-18}$ However, major questions, as discussed below, remain. ${ }^{19-22}$

In many areas of medicine, such as diabetes care or cardiology, clear therapeutic targets have been defined and the continuous effort to reach these targets is standard practice. ${ }^{23-28}$ More recently, treatment targets have also been advocated for rheumatoid arthritis (RA), spondyloarthritides (SpA) including psoriatic arthritis (PsA) and systemic lupus erythematosus, namely remission or low disease activity. ${ }^{29} 30$ These recommendations have been based on insights gained from systematic literature reviews (SLRs) of clinical trials. ${ }^{31} 32$ The successful application of a treat-to-target (T2T) strategy in a rheumatologic disease was first reported in RA and subsequently in PsA. ${ }^{33} 34$

Much less information is currently available regarding the value of defining therapeutic targets for gout. Despite recently published treatment recommendations ${ }^{11-13} 15 \quad 17 \quad 18$ and some scientific and therapeutic advances, such as the approval of the first selective urate transporter inhibitor, a variety of challenges, such as preventing recurrence 
of attacks, remain when considering the current management of patients with gout. These challenges exist, in part, because a clear therapeutic target and strategies to reach such a target have not yet been defined optimally. To improve the management of gout in clinical practice, a core group was convened to discuss and develop consensus recommendations aimed at defining a treatment target and initiate a T2T programme for gout.

\section{METHODS}

Five rheumatologists from Europe and North America with expertise in gout met in Athens in April 2014 and agreed on the need to develop T2T recommendations for gout. A steering committee was established, comprised of rheumatologists who were identified based upon their expertise in gout, experience in developing treatment recommendations, publication record and participation in clinical trials of novel therapies for gout. Through face-to-face discussions and email communications, they agreed to use a four-step methodology:

(i) define treatment targets and outcomes

(ii) perform a systematic literature review (SLR)

(iii) evaluate the findings of the SLR within the steering committee

(iv) develop final recommendations for $\mathrm{T} 2 \mathrm{~T}$ for gout with a larger task force.

Definition of target and outcomes: The steering committee determined potential targets and outcomes by email communication. There was no limitation to the number of targets and outcomes that could be proposed. Each target and outcome was then voted upon by email communication using a Delphi method; those that were supported by at least $75 \%$ of the members were accepted.

Systematic literature review: Search terms for the SLR were formulated based on the PICO (participant intervention, control, outcome) system, taking into account the targets and outcomes (see online supplementary table S1). Authors UK and CF performed the SLR using Medline, EMBASE and Cochrane, focusing on strategy trials in which therapeutic adjustments were made according to prespecified timelines and/or prespecified endpoints. Detailed inclusion and exclusion criteria and the list of search strings are shown in online supplementary tables S1 and S2. All English language human studies were evaluated based on an iterative process with screening of titles, followed by screening of abstract and completed by a full-text review. The results of the SLR were graded for the level of evidence provided by the Oxford Centre for Evidence-Based Medicine on a scale of $1-5 .{ }^{35}$ We did not exclude studies based on quality.

Evaluation: The findings of the SLR were presented to the steering committee at a meeting in Herne, Germany, in June 2015. On this basis, the steering committee formulated a provisional set of recommendations according to European League Against Rheumatism standardised operating procedures fulfilling the AGREE criteria. ${ }^{3637}$ The level of evidence and strength of each recommendation were determined and categorised as A (highest) to D (lowest) on the basis of the SLR. The preliminary recommendations were distributed to the international task force, which consisted of gout experts from North America, South America, Europe, Oceania (New Zealand) and Asia; a nephrologist, a cardiologist, a general practitioner and a patient with gout.

Development of final recommendations: The international multispecialty task force met in Herne, Germany, in October 2015, to discuss the $\mathrm{T} 2 \mathrm{~T}$ approach in detail and develop the final recommendations. The background of the T2T approach was discussed and the preliminary recommendations developed by the steering committee were presented. These were reformulated and reordered, until each recommendation received a majority of at least $75 \%$ of the votes by using a nominal consensus technique. After the face-to-face meeting, the final recommendations were circulated by email to the entire task force for final comments. Only suggestions intended to improve the clarity of wording or to address redundancies were considered; no changes to the content were allowed at this point. The task force then voted by email on the level of agreement with each recommendation using a numerical rating scale of $0-10$, with the highest value indicating the greatest level of agreement.

\section{RESULTS}

Definition of target and outcomes: Six possible treatment targets and five possible outcome domains were identified by the steering committee (table 1). The final list includes four targets and three outcomes about which a SLR should be performed.

Systematic literature review: Of the initial 761 papers retrieved, 55 were selected for full-text review. No randomised trial was identified in which a T2T approach had been evaluated in comparison with standard treatment (flow chart in online supplementary figure S1 illustrates the selection process). However, there was indirect evidence that optimal therapeutic approaches help to control the disease. ${ }^{38-42}$ The investigators showed that control of serum uric acid levels (SUA) is optimised by providing patient education, enhanced telephone access to expert advice, upward titration of allopurinol, febuxostat or benzbromarone and increased frequency of visits until the target SUA concentration was achieved. ${ }^{14}$ Other urate-lowering agents, such as lesinurad, might also be used but strategy trials employing these drugs were not identified in the SLR. Successful reduction of SUA levels by upward titration of ULT, but without prescheduled therapeutic adaptation processes, has been demonstrated in many other trials. ${ }^{38-40} 43 \quad 44$ Patients with gout exhibited a higher velocity of tophus size reduction when reaching a serum urate concentration below the saturation point of MSU early and maintaining that level. ${ }^{39} 4546$

Table 1 Voting on treatment targets and outcomes

\begin{tabular}{lcc}
\hline & Agreement* (\%) & Accept \\
\hline $\begin{array}{l}\text { Treatment targets } \\
\text { Serum urate level }\end{array}$ & 90 & Yes \\
\hline $\begin{array}{l}\text { Time to resolution of inflammation } \\
\text { Amount/reduction/absence of tophi } \\
\text { (burden of deposition) }\end{array}$ & 40 & No \\
\hline $\begin{array}{l}\text { Pain reduction } \\
\text { Prevention/absence of attacks }\end{array}$ & 90 & Yes \\
\hline $\begin{array}{l}\text { Adherence to medication } \\
\text { Outcome }\end{array}$ & 90 & Yes \\
\hline $\begin{array}{l}\text { Clinical: Pain, joint count, number of attacks/year } \\
\text { Laboratory: Serum urate level, CRP, ESR, serum } \\
\text { creatinine }\end{array}$ & 100 & Yes \\
\hline $\begin{array}{l}\text { Functional: SF-36 (physical component score) } \\
\text { Imaging: Radiographs (specific signs), ultrasound } \\
\text { (tophi), dual energy CT (urate deposition) }\end{array}$ & 60 & No \\
\hline $\begin{array}{l}\text { PRO: QoL, SF-36, work status, productivity, work } \\
\text { days off, absenteeism/presenteeism }\end{array}$ & 80 & Yes \\
\hline
\end{tabular}

* 10 members of the steering committee voted.

CRP, C-reactive protein; ESR, erythrocyte sedimentation rate; PRO, patient-reported outcomes; QoL, quality of life; SF-36, short-form 36. 
Significant discussion also focused on asymptomatic hyperuricaemia. Although intervention to treat severe asymptomatic hyperuricaemia has been recommended in Japan, ${ }^{47}$ there is inadequate evidence to support its appropriateness. The task force members agreed that it could be valuable to develop an appropriate strategic approach to the treatment of asymptomatic hyperuricaemia. However, they concurred that there is no evidence to suggest that the treatment target for asymptomatic hyperuricaemia should be any different than that for gout.

Evaluation: Based on the SLR, preliminary recommendations (three overarching principles and eight recommendations) were formulated by the steering committee.

Recommendations: The preliminary recommendations were reformulated and reordered by the task force during a meeting in October 2015, yielding four overarching principles and nine recommendations. The final consensus recommendations are listed in table 2 and discussed in detail below.

\section{Overarching principles}

Since gout is a potentially curable disease, the central guiding principles are to reduce serum urate levels and to maintain a target serum urate level. To achieve these goals, patients must be educated completely about the disease and should participate in the decision-making process. The overarching principles, which are listed in table 2, address the following aspects:

A. Gout as a chronic and serious disease that can be treated effectively. ${ }^{6}$

B. The importance of reducing and maintaining serum urate below a defined target.

C. The requirement for patient education about all aspects of the disease and for shared decision-making with the patient. ${ }^{14}$

D. The need for long-term adherence to urate-lowering therapy.

\section{Recommendations}

The main points discussed were as follows:

(i) Both providers and patients should be educated about the disease and its treatment. (ii) Patients and healthcare providers should make treatment decisions together.

(iii) The primary treatment approach is to reduce and maintain serum urate at a level sufficient to effect dissolution of MSU crystals.

(iv) Modifying lifestyle and monitoring renal function are important.

It was decided to not differentiate between 'acute' and 'chronic' gout, since both belong to the spectrum of a single disease.

All recommendations are presented in table 2 together with the level of supporting evidence, the strength of the recommendation and the level of agreement. Of note and in line with the SLR, the level of evidence was low (D) for most of the recommendations. This lack of evidence supporting how best to treat gout likely is one of the main reasons why it is often difficult to treat the disease successfully and especially to maintain patients free of disease activity over the long term. In the presence of comorbidities, such as renal impairment, the aim of low disease activity is much more complicated to reach which was the basis to recommend assessment of renal function on a regular basis. It also provides the basis for the research agenda, since much more information is needed to develop highly valid treatment recommendations.

Interestingly and despite the low level of evidence, there was a very high level of agreement among task force members, with six of the nine recommendations achieving agreement levels of 9.5 or more on a 10-point scale. This suggests that the experts from different fields and the patient with gout on the task force were quite convinced of the validity of the statements. Only recommendation \#5, which deals with prophylaxis, had a lower level of agreement (8.3). This lower level of agreement was due to the absence of prospectively acquired data regarding the appropriate duration of prophylaxis.

A research agenda was also developed (table 3). It was decided to re-evaluate the current proposals at a future meeting attended by several stakeholders, including more patients. Therefore, the present recommendations should be regarded as a basis for further discussion and amendment.

Table 2 Recommendations to treat-to-target gout

A Gout is a chronic and serious disease with impaired quality of life and reduced life expectancy that can be treated effectively. $9.5 \pm 0.9$

B Reducing and maintaining serum urate below a defined target is mandatory to eliminate urate crystals and improve patient outcomes. $9.8 \pm 0.7$

C Successful management of gout requires education about all aspects of the disease and full involvement of the patient in shared $9.7 \pm 0.7$ decision-making.

D Long-term adherence to urate-lowering therapy is essential for an optimal outcome.

Recommendations

1 Serum urate must be measured regularly and urate-lowering therapy should be adjusted to attain the therapeutic target.

2 A serum urate level $<6 \mathrm{mg} / \mathrm{dL}(<360 \mu \mathrm{mol} / \mathrm{L})$ should be targeted and maintained in all patients with gout

3 In patients with severe gout, such as those with tophi or frequent attacks, the target should be a serum urate level $<5 \mathrm{mg} / \mathrm{dL}(<300 \mu \mathrm{mol} / \mathrm{L}$ ) until clinical remission is achieved

4 Acute attacks should be treated promptly with anti-inflammatory medications, taking safety issues into consideration

5 Prophylaxis against attacks should be initiated and continued for at least 6 months after starting urate-lowering therapy.

6 In all patients with gout, renal function should be assessed at the time of diagnosis and then monitored regularly.

LoE GoR SoR

7 Comorbidities associated with gout may influence therapy and outcomes and should be assessed regularly and managed

8 Modifiable risk factors should be addressed primarily through patient education and support.

9 Information about gout and its management should be made readily available to patients by their healthcare professionals.

SoR on a $0-10$ scale with $0=$ no agreement at all and $10=$ very strong agreement.

GoR, grade of recommendation; LoE, level of evidence; SoR, strength of recommendation. 
Table 3 Research agenda

\section{No Research question}

1 What is the optimal target serum urate level to manage gout?

2 Is the current recommended target serum urate level a valid endpoint?

3 How often should the serum urate level be measured to optimally control disease?

4 How does frequency of serum urate measurement impact on the management of patients with gout?

5 Are very low serum urate levels dangerous (such as by causing neuropsychiatric disease, eg, dementia)?

6 What are the consequences of lowering serum urate levels?

7 Is it possible to develop a composite disease activity measure that might serve as a better target for management of gout disease activity in clinical practice? (GDAl=gout disease activity index).

8 Does an elevated serum urate level cause chronic kidney disease?

\section{$9 \quad$ How often should renal function be measured?}

10 What is the optimal management of gout in patients with multiple comorbidities?

11 Is there any diagnostic utility to aspirating joints in patients with intercritical gout?

12 Should monitoring by ultrasound or CT be used in gout?

13 How is the treatment of both acute and chronic manifestations of gout best integrated into a T2T strategy?

14 What is the optimal timing of initiating treatment for acute gout attacks?

15 What is the optimal strategy to eliminate tophi?

16 Should urate lowering therapy be initiated after the first gout attack?

17 Is there any effect of treating asymptomatic hyperuricaemia?

18 What is the role of surgery in the treatment of tophi?

19 Does a T2T approach improve the adherence of patients to their treatment?

\section{Comment with respect to a T2T approach}

SUA level of $<6 \mathrm{mg} / \mathrm{dL}(<360 \mu \mathrm{mol} / \mathrm{L})$ or $<5 \mathrm{mg} / \mathrm{dL}(<300 \mu \mathrm{mol} / \mathrm{L})$ are not data driven, a valid and reliable cut-off will facilitate a targeted approach.

This important question should be addressed by taking the scientific evidence and the patient point of view into account. A laboratory parameter might not adequately reflect the signs and symptoms of the patient.

Definition of time intervals to measure SUA is mandatory to adapt therapy based on the treatment target.

Frequency of measurements should be feasible and aim to avoid overassessment and underestimation. No trial exists at all to address impact of measurement on the management of patients with gout.

The knowledge about valid upper and lower thresholds of SUA is mandatory to follow a T2T approach.

A T2T approach in patients with gout must address potential harmful consequences (like occurrence of acute attacks while initiating ULT).

More than the SUA level influences disease activity in patients with gout. Therefore, it seems likely that a composite instrument is addressing the patients need more accurate.

The relationship between gout and chronic kidney disease is bidirectional and whether it is a causal relation or an association is still a matter of debate. It still has to be shown that lowering of SUA prevents renal function from further deterioration (and vice versa).

Frequency of measurements should be feasible and aim to avoid overassessment and underestimation.

Determination of treatment target and strategy to adapt the therapy depends on occurrence of many factors, that is comorbidities. Multidisciplinary management should agree upon an achievable treatment target.

The absence of crystals in the synovial fluid is one of the targets that are linked to lowering SUA. Serial joint aspiration studies confirmed the disappearance of crystals with effective ULT. The value of this laboratory finding in patients with absent of gout attacks is questionable.

Diagnostic utility of imaging procedure has been studied to diagnose but has not been extensively studied to monitor the disease. Since deposition of MSU crystals in the tissue is a consequence of increased levels of SUA imaging procedure might serve as a surrogate marker in the management of gout.

Whether the artificial partition between acute and chronic stages is helpful in treating patients with gout is still a matter of debate. However, research on implementations strategies focused on setting a target and adapting the treatment accordingly is strongly needed.

The start of the treatment and prescheduled adaptation process within the treatment strategy is a crucial point that is not yet well defined in studies. Especially, initiating ULT during an acute gout attack remains a matter of debate.

The outcome 'size and number of tophi' is difficult to assess. There is no consensus about the optimal tool to assess this outcome reliable and sensitive to change. However, assessment of tophi is crucial to assess adaptation process within the treatment strategy.

Whether ULT therapy should be initiated after the first gout attack or after a subsequent attack remains controversial. Studies should be conducted to address this question, so as to optimise treatment

Studies are warranted to assess whether T2T approach is also applicable for patients with asymptomatic hyperuricaemia.

Pharmacological interventions are the cornerstone in the T2T approach, but the value of surgical procedures has not been investigated.

Adherence to treatment is low in patients with gout. In a T2T approach, patients do have increased visits at least in the beginning of their disease. One important question is whether this approach is increasing the adherence of patients to their treatment

MSU, monosodium urate; SUA, serum uric acid levels; T2T, target-to-target; ULT, using urate-lowering therapy.

\section{DISCUSSION}

This is the first initiative to propose overarching principles and recommendations for the management of gout using a T2T strategy. Since this approach has been applied to several other rheumatic diseases, ${ }^{29} 30$ we considered it important to employ this successful concept in gout-a disease for which a major target can be defined easily and in which outcomes depend upon maintenance of the serum urate concentration at a low level. However, the scope of the recommendations is focusing on targeted treatment options and on a broader concept including recommendations for prophylaxis against attacks and monitoring of comorbidities. This rationale is based on the fact that aiming for low serum urate concentrations must be supported by antiinflammatory strategies and/or prevention of comorbidities. 
After discussion about asymptomatic hyperuricaemia, the group decided not to make recommendations regarding treatment of hyperuricaemia in the absence of musculoskeletal symptoms because of inadequate evidence. One major concern, which was raised during this discussion, was that treatment targets for asymptomatic hyperuricaemia need to be defined. Further research on this topic is warranted and should be conducted in collaboration with cardiologists and nephrologists.

A T2T approach is based on assessing disease activity or severity. Neither is defined for patients with gout. The term 'severity' is inexact when referring to patients with gout and reflects a variety of parameters, such as frequency of attacks, number of swollen joints, number and size of tophi and presence of comorbidities. No threshold has been defined to differentiate between severe and mild disease states. Some progress has been made in establishing disease activity scores for gout. Recently, the KING study group proposed a new disease activity score for gout including flare, SUA, pain, global activity assessment, joint count and measure of tophi. ${ }^{48}$

However, assessment of outcome domains is not yet standardised in trials with gout patients. Several processes have been implemented within OMERACT to endorse measures of outcome. Pain, joint count and patient global assessment are the recommended outcome measures for patients with acute gout, whereas these three outcome measures with the addition of activity limitation are the outcome measures recommended for patients with chronic gout. ${ }^{49} 50$ There is sufficient evidence to support measures of pain, joint tenderness and swelling as fulfilling the requirements of the OMERACT filter. ${ }^{51}$ However, almost no appreciable impact of the OMERACT-proposed domains could be detected in our SLR. ${ }^{52}$

Initially, we discussed possible treatment targets that should be able to reflect when patients are in remission. The final target list includes pain and SUA levels, as well as the absence of attacks and tophi, as potential parameters to define remission in patients with gout. Remission as a separate variable was not included in our list of treatment targets because no formal decision has yet been made about the set of variables proposed by OMERACT in 2014 to define remission. The publication of preliminary remission criteria for gout in 2016 supports the OMERACT group's recommendation of variables to define remission. $^{53}$

Because of the lack of strategy trials in gout, existing therapeutic recommendations are rather vague. For example, clinicians need guidance regarding the strategic use of anti-inflammatory medications to balance potentially beneficially and harmful effects in patients with acute attacks. However, $\mathrm{T} 2 \mathrm{~T}$ recommendations are primarily generic in nature and do not provide detailed management recommendations or focus on specific drugs. Although some drugs are mentioned as examples, the current recommendations do not endorse one drug over others. With respect to the use of anti-inflammatory medications, our task force was not able to propose a specific sequence of anti-inflammatory medications because trials to address this question were never performed. Therefore, we propose a research agenda to stimulate investigation into the unmet needs in this area, including evaluation of the validity of a T2T strategy in gout. This research agenda includes those questions that were considered to be most important by the task force, but the list obviously could be expanded in many areas, including molecular aspects of pathophysiology and patient and physician considerations.

This represents the first initiative to characterise gout as a disease that should be managed by defining, achieving and maintaining a treatment target to provide patients with the best possible outcome. Its strengths are the inclusion of experts from different areas of medicine and a gout patient representative on the task force and the very high level of agreement that was achieved for most of the recommendations. However, the present activity was limited by the absence of published clinical trials of strategies to treat gout. Thus, recommendations had to be developed based solely upon expert opinion. Another limitation of our approach might be our having limited our SLR to only articles written in the English language. The prevalence of gout and the need for a targeted therapeutic approach is also increasing in less developed countries, such as those in Latin America and in parts of Asia. ${ }^{2}$

Further research is needed to apply our T2T recommendations in a clinical trial, comparing target-directed therapy with routine care. The research agenda addresses many practical questions, such as determining the frequency with which critical blood tests should be performed, assessing the impact of comorbidities and developing a gout disease activity index. Given the low adherence to medication by patients with gout, strategy trials, in which therapeutic adjustments are made according to a prespecified timeline and/or prespecified endpoints, should address this critical issue. Presumably, strategy trials will be able to include an adequate approach to control for non-adherence. ${ }^{54} \mathrm{~A}$ treatment strategy study will be important to improve the management of patients with gout and should be initiated in the near future.

\section{Author affiliations}

${ }^{1}$ Rheumazentrum Ruhrgebiet, and Ruhr University Bochum, Herne, Germany ${ }^{2}$ Division of Rheumatology, Department of Medicine 3, Medical University of Vienna, Vienna, Austria

${ }^{3}$ Assisitance Publique Hôpitaux de Paris Rheumatology Department, Lariboisière Hospital, University Paris Diderot, Sorbonne Paris-Cité and INSERM, UMR 1132, Paris, France

${ }^{4}$ Research Medical Unit INSERM, Université Paris VII—Denis Diderot Assistance Publique-Hôpitaux de Paris, Service de Cardiologie, Hôpital Lariboisière, Paris,

France

${ }^{5}$ University of Auckland and Auckland District Health Board, Auckland, New Zealand

${ }^{6}$ University of Nottingham, Nottingham, UK

${ }^{7}$ Medical Faculty, Institute of General Practice and Family Medicine, University Bonn, Bonn, Germany

${ }^{8}$ UMass Memorial Medical Center and University of Massachusetts Medical School, Worcester, Massachusetts, USA

${ }^{9}$ Carnegie Mellon University, Pittsburgh, Pennsylvania, USA

${ }^{10}$ Rheumatology Division, Hospital de Cruces, Baracaldo, Vizcaya, Spain

${ }^{11}$ Discipline of Rheumatology, Rio de Janeiro State University, Rio de Janeiro, Brazil

${ }^{12}$ University of Alabama at Birmingham, Birmingham, Alabama, USA

${ }^{13}$ Service de Rhumatologie, Centre Hospitalier Universitaire Vaudois and University of Lausanne, Lausanne, Switzerland

${ }^{14}$ Servicio de Reumatología, Hospital General de México, México City, México

${ }^{15}$ Division of Rheumatology, Cedars-Sinai Medical Center, David Geffen School of

Medicine at UCLA, Los Angeles, USA

${ }^{16}$ Medical Department I, Marien Hospital Herne, Ruhr-University of Bochum, Herne, Germany

${ }^{17}$ Tokyo Women's Medical University, Tokyo, Japan

Contributors All authors were fully involved in the T2T project. All authors participated in the voting rounds, meetings and evaluation process. Authors UK and CF did the SLR with judgement by JB. All authors actively participated in writing and revising the paper.

Funding Financial support was requested and obtained from four companies (Novartis, Berlin-Chemie Menarini, Astra-Zeneca and Ardea Bioscience).

Competing interests $J S_{1} M M, B E, J V M, T W, C F$ have no competing interests in respect to this work. UK has received grant and research support and consultancy fees from AbbVie, Chugai, MSD, Novartis, Pfizer, Roche and UCB. JB has received honoraria for talks, advisory boards, paid consultancies and grants from studies from Berlin-Chemie Menarini and Novartis. HY has received honoraria for talks, advisory boards and grants from Abbvie, Astellas, AstraZeneca, BMS, Chugai,

Mitsubishi-Tanabe, Pfizer, Takeda, Teijin and UCB. GRCP has received honoraria for consultancies from: AbbVie, AstraZeneca, BMS Hospira, Janssen, Pfizer, Roche, 
RuiYi, and Sanofi-Aventis. ND has received consulting fees, speaker fees or grants from the following companies: Takeda, Menarini, Teijin, Pfizer, Crealta, Cymabay, Fonterra, Ardea Biosciences and AstraZeneca. MD has received honoraria for ad hoc advisory boards for Ardea Biosciences, AstraZeneca, and Nordic Biosciences. Roche, and AstraZeneca are funding a Nottingham University Investigator-led non-drug study on gout. JK has received research funding paid to the University of Massachusetts Medical School from Ardea Biosciences and consulting fees from AstraZeneca; Novartis Pharmaceuticals Corporation; and Regeneron Pharmaceuticals. KS has received consulting fees and served as a study investigator from Ardea/Astra, Crealta, Takeda. TB has received consulting fees, speaker fees or grants from the following companies: Ipsen Pharma, Menarini, Astrazeneca, Novartis, Sobi and Cymabay.

Provenance and peer review Not commissioned; externally peer reviewed.

\section{REFERENCES}

1 Richette P, Bardin T. Gout. Lancet 2010;375:318-28

2 Kuo CF, Grainge MJ, Zhang W, et al. Global epidemiology of gout: prevalence, incidence and risk factors. Nat Rev Rheumatol 2015;11:649-62.

3 Mikuls TR, Farrar JT, Bilker WB, et al. Gout epidemiology: results from the UK General Practice Research Database, 1990-1999. Ann Rheum Dis 2005;64:267-72

4 Kuo CF, Grainge MJ, Mallen C, et al. Comorbidities in patients with gout prior to and following diagnosis: case-control study. Ann Rheum Dis 2016;75:210-17.

5 Annemans L, Spaepen E, Gaskin M, et al. Gout in the UK and Germany: prevalence, comorbidities and management in general practice 2000-2005. Ann Rheum Dis 2008;67:960-6.

6 Kuo CF, See LC, Luo SF, et al. Gout: an independent risk factor for all-cause and cardiovascular mortality. Rheumatology (Oxford) 2010;49:141-6.

7 Shields GE, Beard SM. A Systematic Review of the Economic and Humanistic Burden of Gout. Pharmacoeconomics 2015;33:1029-47.

8 Pascual E, Sivera F, Andrés M. Synovial fluid analysis for crystals. Curr Opin Rheumatol 2011;23:161-9.

9 van Durme CM, Wechalekar MD, Buchbinder R, et al. Non-steroidal anti-inflammatory drugs for acute gout. Cochrane Database Syst Rev 2014;(9): CD010120.

10 van Echteld I, Wechalekar MD, Schlesinger N, et al. Colchicine for acute gout. Cochrane Database Syst Rev 2014;(8):CD006190.

11 Khanna D, Khanna PP, Fitzgerald JD, et al, American College of Rheumatology. 2012 American College of Rheumatology guidelines for management of gout. Part 2: therapy and antiinflammatory prophylaxis of acute gouty arthritis. Arthritis Care Res (Hoboken) 2012;64:1447-61.

12 Zhang W, Doherty M, Bardin T, et al, EULAR Standing Committee for International Clinical Studies Including. EULAR evidence based recommendations for gout. Part II: Management. Report of a task force of the EULAR Standing Committee for International Clinical Studies Including Therapeutics (ESCISIT). Ann Rheum Dis 2006;65:1312-24.

13 Jordan KM, Cameron JS, Snaith M, et al. British Society for Rheumatology and British Health Professionals in Rheumatology guideline for the management of gout. Rheumatology (Oxford) 2007:46:1372-4.

14 Rees F, Jenkins W, Doherty M. Patients with gout adhere to curative treatment if informed appropriately: proof-of-concept observational study. Ann Rheum Dis 2013;72:826-30

15 Khanna D, Fitzgerald JD, Khanna PP, et al, American College of Rheumatology. 2012 American College of Rheumatology guidelines for management of gout. Part 1: systematic nonpharmacologic and pharmacologic therapeutic approaches to hyperuricemia. Arthritis Care Res (Hoboken) 2012:64:1431-46.

16 Zhang W, Doherty M, Pascual E, et al, EULAR Standing Committee for International Clinical Studies Including. EULAR evidence based recommendations for gout. Part I: Diagnosis. Report of a task force of the Standing Committee for International Clinical Studies Including Therapeutics (ESCISIT). Ann Rheum Dis 2006;65:1301-11.

17 Engel B, Prautzsch H. Management of gout. ZFA 2014;90:7-12.

18 Sivera $\mathrm{F}$, Andrés M, Carmona L, et al. Multinational evidence-based recommendations for the diagnosis and management of gout: integrating systematic literature review and expert opinion of a broad panel of rheumatologists in the $3 \mathrm{e}$ initiative. Ann Rheum Dis 2014;73:328-35.

19 Wise E, Khanna PP. The impact of gout guidelines. Curr Opin Rheumatol 2015;27:225-30.

20 Doherty M, Bardin T, Pascual E. International survey on the diagnosis and management of gout. Ann Rheum Dis 2007;66:1685-6.

21 Khanna PP, FitzGerald J. Evolution of management of gout: a comparison of recent guidelines. Curr Opin Rheumatol 2015;27:139-46.

22 Bardin T, Doherty M. Can we make gout crystal clear? Introduction. Rheumatology (Oxford) 2009;48(Suppl 2):ii1.
23 Conroy RM, Pyörälä K, Fitzgerald AP, et al. Estimation of ten-year risk of fatal cardiovascular disease in Europe: the SCORE project. Eur Heart J 2003;24:987-1003.

24 Warram JH, Manson JE, Krolewski AS. Glycosylated hemoglobin and the risk of retinopathy in insulin-dependent diabetes mellitus. N Eng/ I Med 1995;332:1305-6.

25 Effects of treatment on morbidity in hypertension. II. Results in patients with diastolic blood pressure averaging 90 through $114 \mathrm{~mm} \mathrm{Hg.} \mathrm{JAMA} \mathrm{1970;213:1143-52.}$

26 The effect of intensive treatment of diabetes on the development and progression of long-term complications in insulin-dependent diabetes mellitus.

The Diabetes Control and Complications Trial Research Group. N Eng/ I Med 1993;329:977-86.

27 Egan BM, Lackland DT, Cutler NE. Awareness, knowledge, and attitudes of older Americans about high blood pressure: implications for health care policy, education, and research. Arch Intern Med 2003;163:681-7.

28 Rachmani R, Slavacheski I, Berla M, et al. Treatment of high-risk patients with diabetes: motivation and teaching intervention: a randomized, prospective 8-year follow-up study. J Am Soc Nephrol 2005;16(Suppl 1):S22-6.

29 Smolen JS, Aletaha D, Bij|sma JW, et al. Treating rheumatoid arthritis to target: recommendations of an international task force. Ann Rheum Dis 2010;69:631-7.

30 Smolen JS, Braun J, Dougados M, et al. Treating spondyloarthritis, including ankylosing spondylitis and psoriatic arthritis, to target: recommendations of an international task force. Ann Rheum Dis 2014;73:6-16.

31 Schoels $M$, Knevel $R$, Aletaha $D$, et al. Evidence for treating rheumatoid arthritis to target: results of a systematic literature search. Ann Rheum Dis 2010;69:638-43.

32 Schoels MM, Braun J, Dougados M, et al. Treating axial and peripheral spondyloarthritis, including psoriatic arthritis, to target: results of a systematic literature search to support an international treat-to-target recommendation in spondyloarthritis. Ann Rheum Dis 2014;73:238-42.

33 Coates LC, Moverley AR, McParland L, et al. Effect of tight control of inflammation in early psoriatic arthritis (TICOPA): a UK multicentre, open-label, randomised controlled trial. Lancet 2015;386:2489-98.

34 Grigor C, Capell H, Stirling A, et al. Effect of a treatment strategy of tight control for rheumatoid arthritis (the TICORA study): a single-blind randomised controlled trial. Lancet 2004;364:263-9.

35 Medicine OCfE-b. Levels of Evidence (cited 28 January 2016). http://www.cebm.net/ oxford-centre-evidence-based-medicine-levels-evidence-march-2009/

36 van der Heijde D, Aletaha D, Carmona L, et al. 2014 Update of the EULAR standardised operating procedures for EULAR-endorsed recommendations. Ann Rheum Dis 2015;74:8-13.

37 Brouwers MC, Kho ME, Browman GP, et al. AGREE II: advancing guideline development, reporting and evaluation in health care. J Clin Epidemiol 2010;63:1308-11.

38 Lim AY, Shen L, Tan $\mathrm{CH}$, et al. Achieving treat to target in gout: a clinical practice improvement project. Scand I Rheumatol 2012;41:450-7.

39 Perez-Ruiz F, Calabozo M, Pijoan Jl, et al. Effect of urate-lowering therapy on the velocity of size reduction of tophi in chronic gout. Arthritis Rheum 2002;47:356-60

40 Pascual E, Sivera F. Time required for disappearance of urate crystals from synovial fluid after successful hypouricaemic treatment relates to the duration of gout. Ann Rheum Dis 2007;66:1056-8.

41 Perez-Ruiz F, Alonso-Ruiz A, Calabozo M, et al. Efficacy of allopurinol and benzbromarone for the control of hyperuricaemia. A pathogenic approach to the treatment of primary chronic gout. Ann Rheum Dis 1998;57:545-9.

42 Perez-Ruiz F, Calabozo M, Fernandez-Lopez MJ, et al. Treatment of chronic gout in patients with renal function impairment: an open, randomized, actively controlled study. J Clin Rheumatol 1999;5:49-55.

43 Mitha E, Schumacher HR, Fouche L, et al. Rilonacept for gout flare prevention during initiation of uric acid-lowering therapy: results from the PRESURGE-2 international, phase 3, randomized, placebo-controlled trial. Rheumatology (Oxford) 2013:52:1285-92.

44 Stamp LK, O'Donnell JL, Zhang M, et al. Using allopurinol above the dose based on creatinine clearance is effective and safe in patients with chronic gout, including those with renal impairment. Arthritis Rheum 2011;63:412-21.

45 Sundy JS, Baraf HS, Yood RA, et al. Efficacy and tolerability of pegloticase for the treatment of chronic gout in patients refractory to conventional treatment: two randomized controlled trials. JAMA 2011;306:711-20.

46 Strand V, Khanna D, Singh JA, et al. Improved health-related quality of life and physical function in patients with refractory chronic gout following treatment with pegloticase: evidence from phase III randomized controlled trials. J Rheumatol 2012;39:1450-7.

47 Yamanaka H, Japanese Society of Gout Nucleic Acid Metabolism. Japanese guideline for the management of hyperuricemia and gout: second edition. Nucleosides Nucleotides Nucleic Acids 2011;30:1018-29.

48 Scire CA, Carrara G, Viroli C, et al. Development and first validation of a disease activity score for gout. Arthritis Care Res (Hoboken) 2016; [epub ahead of print 27 Jan 2016]. doi:10.1002/acr.22844. 
49 Singh JA, Taylor WJ, Simon LS, et al. Patient-reported outcomes in chronic gout: a report from OMERACT 10. J Rheumatol 2011;38:1452-7.

50 Singh JA, Taylor WJ, Dalbeth N, et al. OMERACT endorsement of measures of outcome for studies of acute gout. J Rheumatol 2014;41: 569-73.

51 Taylor WJ, Redden D, Dalbeth N, et al. Application of the OMERACT filter to measures of core outcome domains in recent clinical studies of acute gout. J Rheumatol 2014;41:574-80.
52 Araujo F, Cordeiro I, Ramiro S, et al. Outcomes assessed in trials of gout and accordance with OMERACT-proposed domains: a systematic literature review. Rheumatology (Oxford) 2015;54:981-93.

53 de Lautour H, Taylor WJ, Adebajo A, et al. Development of Preliminary Remission Criteria for Gout Using Delphi and 1000Minds Consensus Exercises. Arthritis Care Res (Hoboken) 2016:68:667-72.

54 McGowan B, Bennett K, Silke C, et al. Adherence and persistence to urate-lowering therapies in the Irish setting. Clin Rheumatol 2016;35:715-21. 